
\title{
3 Research Square \\ Trend Analysis of Major Sexually Transmitted Infections in China, 1999-2018
}

Han Chunxia ( $\nabla$ 15731742933@163.com )

Tongji University https://orcid.org/0000-0002-6264-0965

\section{Ai Zisheng}

Tongji University School of Medicine

\section{Research article}

Keywords: sexually transmitted infections, JoinPoint regression, Temporal trend, Annual percentage change (APC), Average annual percentage Change (AAPC)

Posted Date: August 13th, 2020

DOl: https://doi.org/10.21203/rs.3.rs-55956/v1

License: (1) This work is licensed under a Creative Commons Attribution 4.0 International License. Read Full License 


\section{Abstract \\ Background}

Sexually transmitted infections (STIs) are mainly transmitted by sexual contact or similar sexual contact, which can cause diseases of genitourinary organs ,accessory lymphatic system and major organs of the whole body. STIs include clinically symptomatic diseases and asymptomatic infections. The World Health Organization (WHO) reports that More than 1 million sexually transmitted infections (STIs) are acquired every day worldwide.By analyzing the temporal trend of the sexually transmitted infections incidence in China in the past 20 years, we can provide a scientific basis for the further development of prevention and control measures related to sexually transmitted infections.

\section{Methods}

Joinpoint regression model is used to fit the incidence data of three sexually transmitted diseases in China from 1999 to 2018. Annual percentage change (APC) and Average annual percentage Change (AAPC) are calculated to evaluate the temporal trend changes of the incidence of three major sexually transmitted diseases.

\section{Results}

The overall incidence of AIDS/HIV has been on the rise for 20 years, rising by an average of $33.7 \%$ per year $(P<0.05)$. The most obvious increase is in $2002-2005$, an average annual increase of $102.5 \%$. The overall incidence of gonorrhea is on a downward trend, decreasing by $4.9 \%$ per year on average $(P<$ 0.05).However, its incidence shows a slow rising trend from 2012 to 2018, with an average annual increase of $6.0 \%$. The overall incidence of syphilis is also on the rise, increasing by an average of $9.1 \%$ per year $(P<0.05)$. Except 1999-2003, the incidence of syphilis shows a downward trend, and the other time stages shows a significant upward trend.

\section{Conclusions}

In the past 20 years, the corresponding measures have been taken in China for sexually transmitted infections and achieved remarkable results, but the three major sexually transmitted infections are still in the forefront of the reported notifiable infectious diseases incidence in China. At the same time, all sexually transmitted infections are beginning to shift from high-risk population to the general population, especially adolescents and the floating population. Therefore, China should still pay more attention to the prevention and control of STIs to hold back their further spread or epidemic.

\section{Background}


Sexually transmitted infections (STIS) STIs are mainly transmitted by sexual contact or similar sexual contact, which can cause diseases of genitourinary organs, accessory lymphatic system and major organs of the whole body. STIs include clinically symptomatic diseases and asymptomatic infections. The World Health Organization (WHO) reports that More than 1 million sexually transmitted infections (STIs) are acquired every day worldwide[1]. Most STIs have no symptoms or only mild symptoms that may not be identified as STIs. In some cases, STIs can not only harm the patients, but can also lead to stillbirth, neonatal death, low birth weight and premature birth, sepsis, pneumonia, neonatal conjunctivitis and even congenital malformations through mother-to-child transmission. Thus it can be seen that STIs is a worldwide social and public health problem, and is one of the main diseases harmful to people's reproductive health.

The JoinPoint regression model, also known as fragment regression, was proposed by Kim in $2000[2]$.The basic idea is to divide a long-term liner trend into several segments, each of which is described by a continuous linear pattern. It mainly includes two models: linear data model and logarithmic linear data model. The former is mainly used for the data of dependent variables following normal distribution or approximate normal distribution, while the latter is used for the data of dependent variables obeying Poisson distribution or exponential distribution. Because the JoinPoint regression model does not have strict requirements on whether there is a trend in the data itself, which is often used to study the epidemiological trends in cancer and chronic diseases[3-5].

Currently, no studies have used the JoinPoint regression model to analyze the incidence trends of major sexually transmitted infections in China over the past 20 years. The purpose of this study is to analyze the temporal trend changes of the incidence of sexually transmitted infections (STIs) through the Jointpoint regression model, so as to provide a basis for the further development of prevention and intervention measures related to STIs and control the further epidemic of STIs in China.

\section{Methods}

\section{Data sources}

The data are obtained from the incidence data of HIV/AIDS, syphilis and gonorrhea in the Chinese Health Statistics Yearbook (2003-2019)[6] .The data unit is 1/100,000. (Table 1)

\section{Table 1 Incidence of HIV, syphilis, gonorrhea in China in 1999-2018}




\begin{tabular}{|llll|}
\hline year & Incidence of AIDS & $\begin{array}{l}\text { Incidence of } \\
\text { syphilis }\end{array}$ & $\begin{array}{l}\text { Incidence of } \\
\text { gonorrhea }\end{array}$ \\
\hline & $\begin{array}{c}(1 / 100,000 \square \\
(1 / 100,000)\end{array}$ & $(1 / 100,000)$ \\
\hline 2000 & 0.02 & 6.50 & 27.54 \\
\hline 2001 & 0.04 & 6.43 & 22.92 \\
\hline 2002 & 0.06 & 6.11 & 18.57 \\
\hline 2003 & 0.08 & 5.80 & 16.14 \\
\hline 2004 & 0.23 & 5.63 & 16.54 \\
\hline 2005 & 0.43 & 7.70 & 17.71 \\
\hline 2006 & 0.60 & 10.96 & 14.27 \\
\hline 2007 & 0.82 & 14.24 & 12.46 \\
\hline 2008 & 1.10 & 17.16 & 11.33 \\
\hline 2009 & 1.51 & 21.06 & 10.16 \\
\hline 2010 & 2.56 & 24.66 & 9.19 \\
\hline 2011 & 2.92 & 28.90 & 8.07 \\
\hline 2012 & 3.11 & 32.04 & 7.61 \\
\hline 2013 & 3.12 & 33.30 & 7.07 \\
\hline 2014 & 3.33 & 32.86 & 7.61 \\
\hline 2015 & 3.69 & 30.93 & 7.05 \\
\hline 2016 & 3.97 & 31.85 & 7.36 \\
\hline 2017 & 4.15 & 35.63 & 9.59 \\
\hline 2018 & 4.62 & & 8.39 \\
\hline & & 31.97 & 10.06 \\
\hline
\end{tabular}

\section{Basic index}

Due to the absence of morbidity and mortality data in some years in the yearbook, only complete morbidity data of three STIs are used in this study.

\section{Joinpoint regression analysis}

The data analysis is performed using the Joinpoint Regression Software (4.8.1.0 version developed by the American cancer research center). The software can analyze and fit the data on the incidence of three 
sexually transmitted infections by logarithmic linear model. The number, location and the corresponding $P$ value of the connection points were determined by the monte carlo permutation test[2, 4]. Since the number of observed values is only 20 , the default number of connection points in the software is at most 3. $P<0.05$ was statistically significant.

The Joinpoint regression model is used to fit the incidence of HIV/AIDS, syphilis and gonorrhea respectively, and annual percent Change (APC) and average annual Percent Change (AAPC) are calculated to evaluate the temporal trend changes of the incidence of the three major sexually transmitted infections (STIs) in China from 1999 to 2018. The model will be fitted to analyze the change of three STIs in different years. If $A P C<0$, indicating that the incidence rate is decreasing year by year; If $A P C>0$, meaning that the incidence of disease decreases year by year ; If APC=AAPC, indicating no connection points and this group of data increases monotonously or decreases monotonously with the change of the years.

\section{Results}

\section{Trend analysis of AIDS/HIV incidence}

As can be seen from Figure 1, the temporal trend changes of AIDS/HIV incidence have three connection points from 1999 to 2018. They are in 2002,2005 and 2011 respectively. The overall incidence of AIDS has been on the rise for 20 years, rising by an average of 33.7\% per year $(P<0.05)$. From 1999 to 2002, the APC shows a slowly rising trend, with 42.6\%; And a rapid upward trend in 2002-2005, APC is up to $102.5 \%$; But there is a slow upward trend again in 2005-2011, APC is $38.2 \%$; At the same time, the upward trend from 2011 to 2018 further slow down, APC is $5.7 \%$ (Table 2).

Table 2 APC and AAPC of AIDS incidence in Chinese population ,1999-2018

\begin{tabular}{|lll|}
\hline year & APC/\% & AAPC/\% \\
\cline { 1 - 2 } $1999-2002$ & $42.62^{\star}$ & $33.7^{*}$ \\
\cline { 1 - 2 } $2002-2005$ & $102.52^{\star}$ & \\
\cline { 1 - 2 } $2005-2011$ & $38.25^{\star}$ & \\
\cline { 1 - 2 } $2011-2018$ & $5.74^{\star}$ & \\
\hline
\end{tabular}

* $P<0.05$

\section{Trend analysis of gonorrhea incidence}

The results of gonorrhea incidence are shown in Figure 2. The temporal trend changes of gonorrhea incidence from 1999 to 2018 have only 1 connection point, which is in 2012. The overall incidence of gonorrhea has been declining for 20 years, by an average of $4.9 \%$ per year $(P<0.05)$.From 1999 to 2012 , 
there is a rapid downward trend, with APC of $-9.6 \%$; However, it shows a slow upward trend from 2012 to 2018 , with APC of $6.0 \%$ (Table 3).

Table 3 APC and AAPC of gonorrhea incidence in Chinese population ,1999-2018

\begin{tabular}{|lll|}
\hline year & APC/\% & AAPC/\% \\
\cline { 1 - 2 } $1999-2012$ & $-9.57 *$ & $-4.9 *$ \\
\cline { 1 - 2 } $2012-2018$ & $5.96 *$ & \\
\cline { 1 - 2 } & &
\end{tabular}

* $P<0.05$

\section{Trend analysis of syphilis incidence}

As shown in Figure 3, there are 3 connection points in the temporal trend changes of syphilis incidence from 1999 to 2018, and the number of connection points is consistent with HIV/AIDS, respectively in 2003, 2006 and 2010.The overall incidence of syphilis shows an upward trend, with an average annual increase of $9.1 \%(P<0.0001)$. The APC shows a downward trend from 1999 to 2003 , which is $-3.6 \%$; From 2003 to 2006 , there is an upward trend, with APC of $36.6 \%$; Although the two time periods of 2006-2010 and $2010-2018$ remain on the rise, APC is $20.3 \%$ and $1.7 \%$ respectively, which is significantly lower than that in 2003-2006(Table 4).

Table 4 APC and AAPC of syphilis incidence in Chinese population ,1999-2018

\begin{tabular}{|lll|}
\hline year & APC/\% & AAPC/\% \\
\cline { 1 - 2 } $1999-2003$ & $-3.65^{\star}$ & $9.1^{\star}$ \\
\cline { 2 - 2 } $2003-2006$ & $36.62^{\star}$ & \\
$2006-2010$ & $20.33^{\star}$ & \\
\cline { 1 - 2 } $2010-2018$ & $1.68^{\star}$ & \\
\hline
\end{tabular}

$\star P<0.05$

\section{Discussion}

The results of this study show that the incidence of AIDS/HIV in Chinese population increased year by year from 1999 to 2018,which are consistent with the results of Yong-Chao and Zunyou Wu's study[7, 8]. The rising tendency of morbidity is most pronounced during the period of 2002-2005, the average annual increase was $102.5 \%$. The possible reasons are the abuse of intravenous drug , the increase in the number of homosexual men and infection occurred after irregular blood collection in this time stage[9]. However, since the implementation of the blood donation law in 1998, the transmission of HIV through blood collection and supply has been basically controlled in China. At present, the transmission of HIV is 
mainly through sexual transmission[10,11]. The introduction of "four exemptions and one care" policy in 2006 and the implementation of the "six expansion and five strengthening" prevention and control measures in 2011 have also effectively slowed down the rising tendency of AIDS incidence, which is consistent with the results of our study[12-14]. At the same time, a research report on the global AIDS epidemic shows that compared with other countries, the current AIDS epidemic in China is at a low epidemic level[15]. But the relevant studies indicate that the AIDS infected people in China are changing from high-risk population to general population, especially adolescents and the floating population [7, 16]. Adolescents not only have a high incidence of premarital sex but also have multiple sexual partners, leading to a high incidence of sexually transmitted infections among adolescents. According to the survey, 1 out of 7 new HIV infections in the world now occur in adolescence, AIDS has become the second leading cause of death among adolescents worldwide[16]. At the same time, due to the low education level of the floating population, their knowledge, attitudes and behaviors of HIV are limited, and their strong mobility leads to the floating population becoming a bridge for the spread of HIV $[9,17]$.Therefore, in order to prevent the epidemic of AIDS in the general population, our country should strengthen the sex education, health promotion and health education for the two groups of teenagers and floating population .

Gonorrhea is reported to be the most common sexually transmitted infection in any country in the world[18, 19], with 106 million new cases of gonorrhea in adults worldwide each year and extremely high exposure rates. Not only is it a major cause of pelvic inflammatory disease and infertility in women, it can also increase the sexual transmission and prevalence of HIV.Therefore, the gonorrhea has a significant impact on reproductive health. The epidemic of gonorrhea in China rose rapidly in the 1970s, reached its peak in 1999, and then declined, which is consistent with the results of our study[20]. But our study shows that the incidence of gonorrhea showed a significant decrease in 1999-2012,and then showed a significant increase from 2012 to 2018. The increased trend of the gonorrhea incidence in China from 2012 to 2018 is similar to that of the United States and the United Kingdom in the same years[21].An increase of the gonorrhea incidence at this time stage in China may be due to the expanded coverage of gonorrhea screening,the increased sensitivity of diagnostic methods and the increase in the number of MSM patients[22, 23]. Although this study indicates that the incidence of gonorrhea in China from 1999 to 2018 shows an overall trend of decline, it is still in the forefront of the notifiable infectious diseases. Especially in 2018 its incidence is $9.58 \%$, ranking fourth[6]. Gonorrhea is currently treated with antibiotics because there is no vaccine that can be prevented. However, neisseria gonorrhoeae has been found to be increasingly resistant to many antibiotics, making the treatment of gonorrhea become a major challenge for clinicians in recent years $[11,19,24,25]$. Therefore, in order to control the rising trend of gonorrhea epidemic in China, the state not only needs to actively publicize the knowledge related to gonorrhea among high-risk population, so as to enhance the public awareness of prevention and treatment of gonorrhea, but also needs to increase the research and development of gonorrhea related vaccines and drugs.

According to the reports of WHO, 988000 pregnant women were infected with syphilis in 2016, resulting in over 350000 adverse birth outcomes including 200000 stillbirths and newborn deaths[26]. It can be 
seen that syphilis seriously affects the life and health of women who are in childbearing age and of newborns[27]. The results of our study show that the incidence of syphilis in China is on the rise from 1999 to 2018 on the whole, which is contrary to the overall temporal trend of gonorrhea incidence[20]. The trends in the incidence of both sexually transmitted diseases are similar to developed countries. On the one hand, this phenomenon may be due to the increased number of syphilis infection population in MSM of China; On the other hand, it may be because the Government launched a national plan on expanding syphilis screening in 2010, which led to an increase in the number of cases diagnosed as latent syphilis[28, 29]. And the related literature also suggest that syphilis infection rate is higher than AIDS in Chinese MSM population [7]. From the four temporal stages of syphilis incidence, we can learn that syphilis incidence has a downward trend from 1999 to 2003, the rest of time stages presents a steady upward trend. It also suggests that China should pay more attention to the prevention and treatment of syphilis. At the same time, the relevant studies have shown that the majority of Chinese people's knowledge of syphilis is far lower than the standard of 10-year plan set by the our country, which requires the awareness rate of the general population reaches $90 \%$ and that of high-risk population reaches $95 \%$ by $2020[27,28,30]$. Therefore, the country should strengthen the publicity of syphilis prevention knowledge, enhance the prevention and control ability and prevent its further spread.

\section{Conclusion}

To sum up, our country has taken corresponding prevention and control measures for AIDS, gonorrhea, syphilis and other sexually transmitted diseases, and achieved good results in the past 20 years. But the Chinese Health Statistics Yearbook (2019) shows that the incidence of three kinds of sexually transmitted infections is still in the forefront of the notifiable infectious diseases[6]. At the same time, the precocious puberty of teenagers generally leads to the increase in the proportion of premarital sex. In addition, most teenagers have multiple sexual partners, These factors lead to teenagers become the high-risk population of sexually transmitted infections[16]. Therefore, the prevention and control of sexually transmitted infections (STIs) in China needs to provide widely recognized effective prevention, screening, diagnosis and treatment interventions for centers for Disease Control and Prevention and hospitals at all levels. In addition, it is necessary to actively popularize knowledge related to gonorrhea and syphilis, and carry out large-scale peer education[31], so as to further reduce the spread or epidemic of sexually transmitted infections in China.

\section{Abbreviations}

STIS: Sexually transmitted infections

HIV: Human immunodeficiency virus

AIDS: Acquired immune deficiency syndrome

APC: Annual percentage change 
AAPC: Average annual percentage change

\section{Declarations}

Acknowledgements

No Applicable.

Funding

None.

Contributions

All authors contributed to the manuscript proof. All authors read and approved the final manuscript.

Ethics declarations

Consent for publication

No Applicable.

Competing interests

The authors declare that they have no competing interests.

\section{References}

1. Yin M, Guo Q: A million people a day get sexual infections, WHO warns \%J Chinese Journal of Infection and Chemotherapy. 2020, 20(02):180.

2. Kim HJ, Fay MP, Feuer EJ, Midthune DN: Permutation tests for joinpoint regression with applications to cancer rates. Statistics in medicine 2000, 19(3):335-351.

3. Ceng S: Joinpoint regression model and its application in epidemic trend analysis of infectious diseases \%J Chinese Journal of Health Statistics. 2019, 36(05):787-791.

4. Zhou W, Zhang Z, Bi Y, Wang L, Liu X, Yu C: Joinpoint regression analysis for the mortality trend of breast cancer in Chinese female from 1987 to2014\%J Journal of Centeral South University(Medical Science). 2018, 43(02):210-215.

5. Arfe A, Malvezzi M, Bertuccio P, Decarli A, La Vecchia C, Negri E: Cancer mortality trend analysis in Italy, 1970-2007. Eur J Cancer Prev 2011, 20(5):364-374.

6. picture "china health statistics yearbook" editorial board. In., vol. 978-7-81136-740-9: P4.

7. Qiao YC, Xu Y, Jiang DX, Wang X, Wang F, Yang J, Wei YS: Epidemiological analyses of regional and age differences of HIV/AIDS prevalence in China, 2004-2016. Int J Infect Dis 2019, 81:215-220. 
8. Wu Z, Chen J, Scott SR, McGoogan JM: History of the HIV Epidemic in China. Curr HIV/AIDS Rep 2019, 16(6):458-466.

9. Wang X, Lan G, Shen Z, Vermund SH, Zhu Q, Chen Y, Khoshnood K, Wu Z, Tang Z: HIV and syphilis prevalence trends among men who have sex with men in Guangxi, China: yearly cross-sectional surveys, 2008-2012. BMC infectious diseases 2014, 14:367.

10. Wang L, Santella AJ, Wei X, Zhuang G, Li H, Zhang H, Huang X, Zhang Y, Zheng H, Zhou A: Prevalence and protective factors of HIV and syphilis infection among men who have sex with men in Northwest China. J Med Virol 2019.

11. Xiu L, Yuan Q, Li Y, Zhang C, Tang L, Peng J: Emergence of ceftriaxone-resistant Neisseria gonorrhoeae strains harbouring a novel mosaic penA gene in China. The Journal of antimicrobial chemotherapy 2020, 75(4):907-910.

12. Yan J, Chen Q, Ma Y, Suo L, Cohen M: Analysis for development and impacting factors about HtIV/AIDS policy in China \%J Chinese Journal of Epidemiology. 2005(11):27-32.

13. Zheng L, Chen Q, Shen J: China's AIDS prevention and control policy and strategy development process backtrack \%J Chinese Journal of AIDS \& STD. 2019, 25(07):657-661.

14. Zheng X: To curb the spread of AIDS in China through drug use and blood supply \%J Chinese Journal of Epidemiology. 2000(01):7.

15. Frank TD, Carter A, Jahagirdar D, Biehl MH, Douwes-Schultz D, Larson SL, Arora M, Dwyer-Lindgren L, Steuben $\mathrm{KM}$, Abbastabar $\mathrm{H}$ et al: Global, regional, and national incidence, prevalence, and mortality of HIV, 1980-2017, and forecasts to 2030, for 195 countries and territories: a systematic analysis for the Global Burden of Diseases, Injuries, and Risk Factors Study 2017. The Lancet HIV 2019, 6(12):e831-e859.

16. Zhang Y, Zhang X, Li X, Gu X: Status of adolescent reproductive health\%J Chinese Journal of Family Planning \& Gynecotokology. 2020, 12(04):4-6.

17. Li S, Li F, Wang H, Liu H, Guo S, Hou Z: Monitoring and analysis of AIDS and STD among floating population at construction site in Changping District of Beijing from 2016-2018\%J Occupation and Health 2019, 35(24):3378-3381.

18. Bala M, Kakran M, Singh V, Sood S, Ramesh V: Monitoring antimicrobial resistance in Neisseria gonorrhoeae in selected countries of the WHO South-East Asia Region between 2009 and 2012: a retrospective analysis. Sexually transmitted infections 2013, 89 Suppl 4:iv28-35.

19. Ma J, Li G, ZHang Y: Analysis of drug resistance of Neisseria gonorrhoeae \%J Chinese Journal of Human Sexuality. 2015, 24(06):70-72.

20. Ma J, Liu N, Chen A, Yang G: Study on knowledge, attitudes and behaviors regarding infectious diseases among Chinese people in 2002 \% J Chinese Journal of Epidemiology. 2005(06):389-393.

21. Rice PA, Shafer WM, Ram S, Jerse AE: Neisseria gonorrhoeae: Drug Resistance, Mouse Models, and Vaccine Development. Annual review of microbiology 2017, 71:665-686.

22. Wang C, Tang W, Zhao P, Tucker J, Chen L, Smith MK, Wong NS, Dong W, Yang B, Zheng H: Rapid increase of gonorrhoea cases in Guangdong Province, China, 2014-2017: a review of surveillance 
data. BMJ Open 2019, 9(11):e031578.

23. Liu C, Hou S, Wang H, Cheng S, Hu X, Zheng W, Zhang X, Chen X: Epidemiological characteristics of gonorrhea in Huangshan City from 2014 to 2018 \%J Chinese Journal of School Doctor. 2020, 34(04):257-258+279.

24. Shigemura K, Fujisawa M: History and epidemiology of antibiotic susceptibilities of Neisseria gonorrhoeae. Current drug targets 2015, 16(3):272-280.

25. Guidelines for diagnosis and treatment of syphilis, gonorrhea and genital Chlamydia trachomatis infection $\mathbf{2 2 0 2 0}$. Chinese Journal of Dermatology 2020, 53(3).

26. Korenromp EL, Rowley J, Alonso M, Mello MB, Wijesooriya NS, Mahiané SG, Ishikawa N, Le LV, Newman-Owiredu $\mathrm{M}$, Nagelkerke $\mathrm{N}$ et al: Global burden of maternal and congenital syphilis and associated adverse birth outcomes-Estimates for 2016 and progress since 2012. PloS one 2019, 14(2):e0211720.

27. Tucker JD, Cohen MS: China's syphilis epidemic: epidemiology, proximate determinants of spread, and control responses. Curr Opin Infect Dis 2011, 24(1):50-55.

28. Wu X, Hong F, Lan L, Zhang C, Feng T, Yang Y: Poor awareness of syphilis prevention and treatment knowledge among six different populations in south China. BMC public health 2016, 16:287.

29. Chen XS: Syphilis trends in China: biased by screening and reporting? The Lancet Infectious diseases 2017, 17(8):804.

30. Ying L, Shaokai T, Xingdong Y, Wanping H, Jinliang L, Qian Y, Kexian G: Awareness and knowledge of syphilis among different populations in Guangzhou, Guangdong Province, China. Sexual health 2013, 10(3):282-283.

31. He J, Wang Y, Du Z, Liao J, He N, Hao Y: Peer education for HIV prevention among high-risk groups: a systematic review and meta-analysis. BMC infectious diseases 2020, 20(1):338.

\section{Figures}



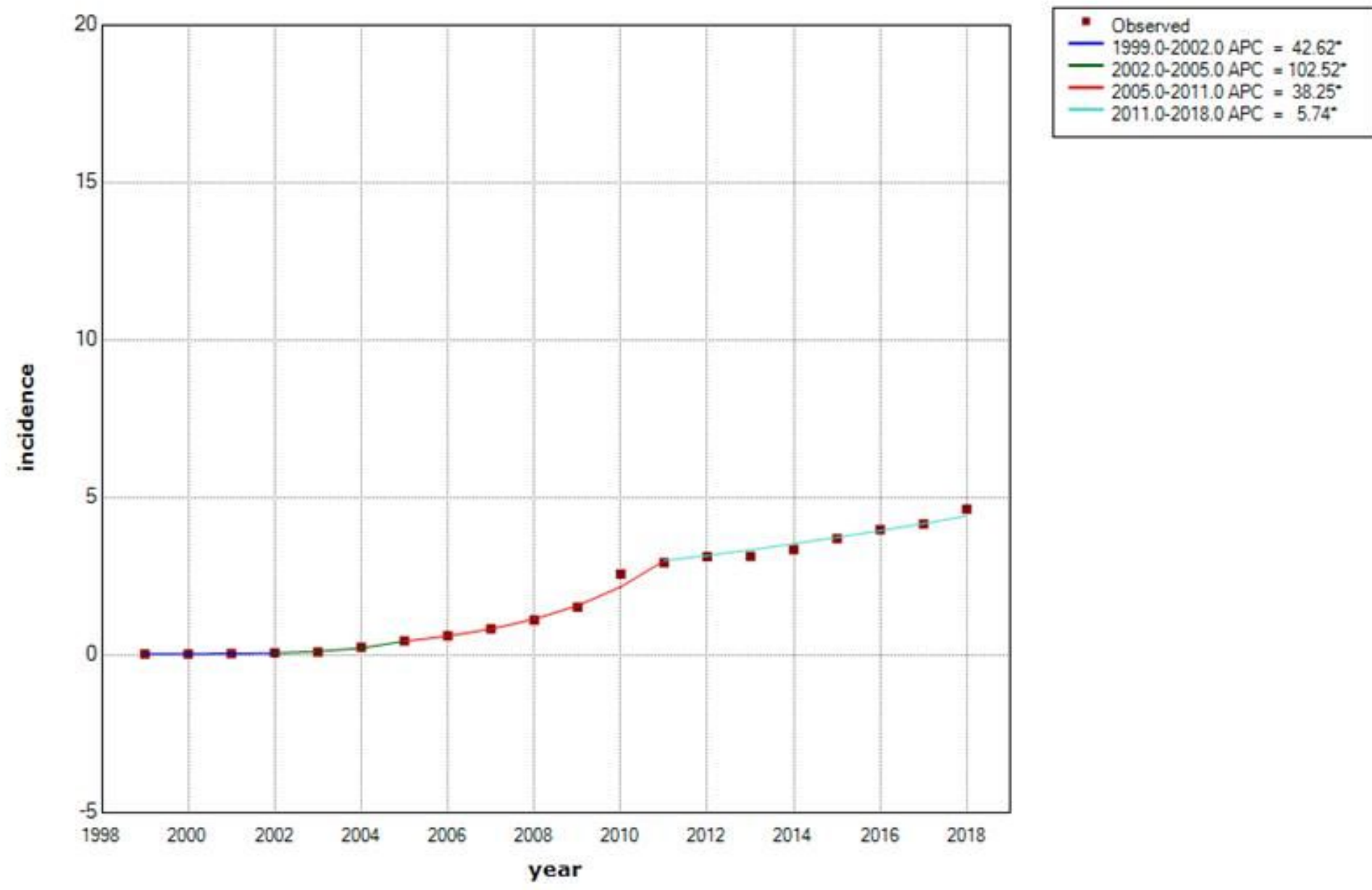

Figure 1

Time trend of AIDS/HIV incidence in Chinese population ,1999-2018 * indicates that the Annual Percent Change (APC) is significantly different from zero at the alpha $=0.05$ level. Final selected model: 3 joinpoints. 


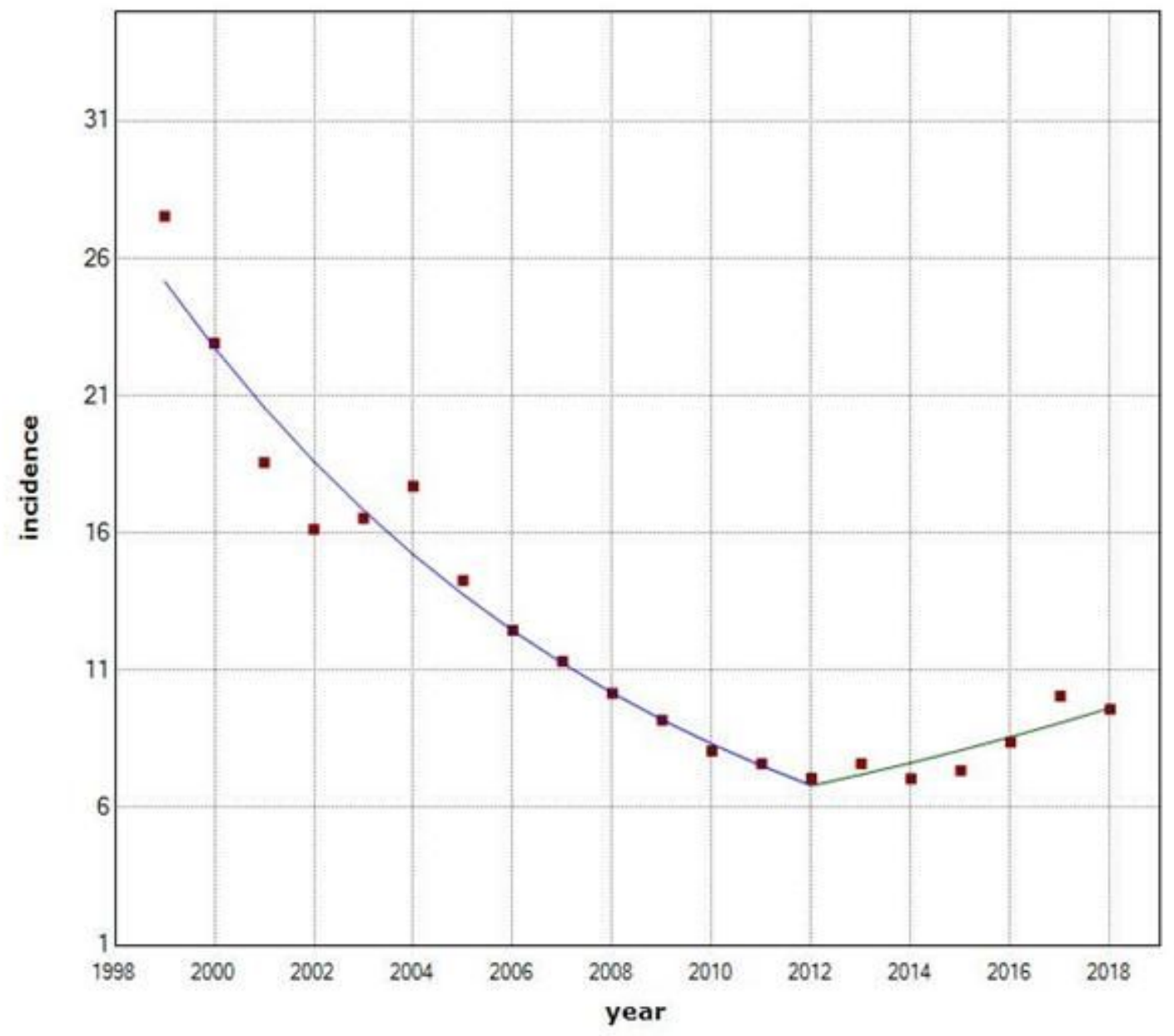

Figure 2

Time trend of gonorrhea incidence in Chinese population ,1999-2018. * indicates that the Annual Percent Change (APC) is significantly different from zero at the alpha $=0.05$ level. Final selected model: 2 joinpoints. 


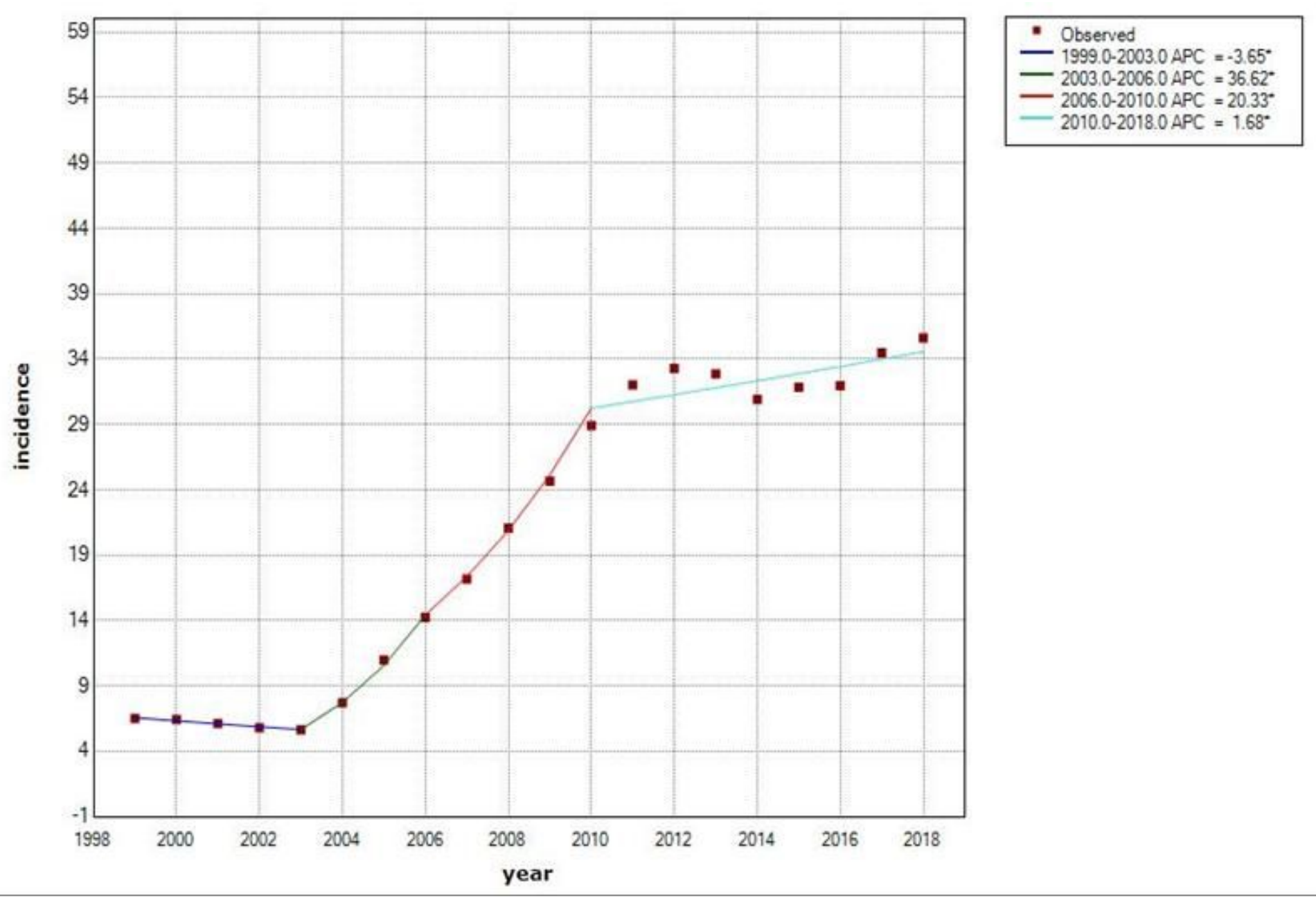

\section{Figure 3}

Time trend of syphilis incidence in Chinese population ,1999-2018. * indicates that the Annual Percent Change (APC) is significantly different from zero at the alpha $=0.05$ level. Final selected model: 4 joinpoints. 